\title{
Introduction: High-Grade Gliomas
}

February 2003

By Isabelle M. Germano, M.D.

The management of high-grade gliomas remains one of the most challenging issues in neurosurgery. High-grade gliomas are the most frequent primary brain tumors in adult patients. Although primary malignant astrocytomas constitute only approximately $2 \%$ of all cancers, they are associated with severe disability and high mortality rate. Patients harboring high-grade gliomas are often affected in the most productive years of their lives. In the pediatric population, although high-grade gliomas occur less frequently than in the adults, they are associated with high rates of mobidity and mortality.

The pioneers of brain tumor surgery, including Cushing, Dandy, Keen, Macewen, and Horsley, demonstrated the possibility of performing brain tumor surgery in the beginning of the 20th century. Over the past two decades, technological advancements have allowed us a better understanding of many aspects of brain tumors, including cell biology and molecular events, and have facilitated the development of equipment necessary for refined neurosurgical intervention. It is now well accepted that the ideal management of brain tumors involves a multidisciplinary approach involving the best technical, scientific, and human resources of a medical center.

This issue of Neurosurgical Focus reflects the challenges and advances in treatment of high-grade gliomas. The most innovative topics in neurooncology are addressed in this issue including but not limited to advances in molecular biology, chemotherapy involving delivery by convention-enhanced techniques, and image-guided technology. In the first paper, Tamber, et al., highlight some of the most important concepts in management of pediatric supratentorial high-grade gliomas. Novel approaches are reviewed and discussed. Hall, et al., review a timely topic that is perhaps one of the more novel innovations in malignant glioma therapy. The high-flow microinfusion delivery technique, known as convection-enahnced delivery, is used to overcome the problem of the blood::brain barrier when delivering chemotherapy. Although clinical experience is limited, microfusion seems to be a very promising therapeutic adjunct in the treatment of high-grade gliomas.

Perhaps one of the most important aspects of management of high-grade gliomas is the constant trial of new chemotherapeutic agents that might target tumor cells while sparing normal tissue. Two groups in this issue provide clinical and laboratory results of promise. Briceno, et al., report on a remarkable series in which survival advantages were observed in patients treated with chloroquine and carmustine compared with those who received carmustine alone. Although therapy was not administered empirically, the results are impressive and promising. Rami, et al., report on extensive testing on okadaic acid, a novel agent with potential therapeutic effects against selected glioma cells.

Finally, the limitations and advances in frameless image-guided surgery for high-grade gliomas are discussed in the last two papers. Benveniste and Germano evaluate factors predictive of brain shift during image-guided resection of suptatentorial malignant gliomas. Nikas, et al., describe the interface of two commonly used image-guided systems: intraoperative ultrasonography and preoperative magnetic resonance imaging. The comparison of these two methodologies allows compensation for intraoperative brain shift.

This is an inspiring time for neurosurgeons caring for patients with high-grade gliomas. Although we are still far from able to provide a cure, the hard work of individuals dedicated to this field continues to allow its advancement.

Neurosurg. Focus / Volume 14 / February, 2003 